\title{
Efficacy of Hayman Suture Technique in the Management of Atonic Post-Partum Hemorrhage
}

\author{
ANAMIKA MAJUMDAR ${ }^{1}$, KALLOL MALLICK $^{2}$, BIPIN VASAVA $^{2}$, KANAN T. DESA $^{3}$
}

\begin{abstract}
Objective: To study the effectiveness of Hayman suture technique to control postpartum hemorrhage.

Method: Hayman suture was applied in 43 cases. A No-1 Vicryl suture was used on a round body or a straight needle. In 36 cases (83.72\%) Hayman suture was the only intervention. In 4 cases (9.30\%) uterine vessels and ovarian vessels were also ligated along with Hayman suture. In 3 cases (7\%), along with Hayman suture, Gunashila's circumferential sutures were taken.
\end{abstract}

Results: None of the 43 patients required hysterectomy.

Conclusion: Hayman suture technique is an easily and rapidly applied, effective, simple, safe, life saving and fertility preserving method to control atonic postpartum hemorrhage.

Keywords: Atonic Post Partum Hemorrhage, Hayman Suture, B-Lynch Suture.

\section{Introduction:}

It has been estimated that world wide over 125000 women die of postpartum hemorrhage (PPH) each year $^{1}$. PPH complicates approximately $3.7 \%$ of vaginal and $6.4 \%$ of caesarean delivery ${ }^{2}$. In India $25.6 \%$ of maternal deaths are due to hemorrhage ${ }^{3}$. Uterine atony accounts for $75 \%-90 \%$ of primary $\mathrm{PPH}$. The traditional management of this condition begins with conservative methods such as bimanual compression, medical therapy with uterotonic agents, uterine temponade with balloons and occasionally arterial embolisation ${ }^{4}$, the failure of which often mandates surgical intervention. Surgical measures such as ligation of the major pelvic vessels demand a rarely used skill possessed by few registrars. In the event of intractable hemorrhage, despite the above measures, hysterectomy is usually the final resort.

In 1997, Christopher B- Lynch devised an innovative technique to treat uterine atony, where a continuous suture was used to envelop and mechanically compress the uterus in an attempt to avoid hystrectomy ${ }^{4}$. Hayman suture, the modification of B- Lynch suture, offers the potential advantage that it can be applied faster and easier, avoiding the performance of a lower segment hysterectomy when $\mathrm{PPH}$ follows a vaginal delivery ${ }^{5}$. Our study reviews the cases in which the Hayman suture was used in our institution to treat primary atonic PPH and their clinical outcomes are discussed.

\section{Materials and methods:}

A retrospective study of all women who delivered between April, 2007 and March, 2010 was collected from our departmental database. Primary $\mathrm{PPH}$ is defined as a blood loss of more than $500 \mathrm{ml}$ at or with in 24 hours of delivery. There were a total of 18,750 deliveries during this period, with primary $\mathrm{PPH}$ occurring in 938 of these cases. The Hayman suture procedure was performed in 43 cases only after uterine atony did not respond to measures such as uterine massage, bimanual compression and the use of uterotonics, i.e. Oxytocin, Ergometrine, PG-F2á, Misoprostol. Out of these 43 cases, 38 cases delivered in our institute and 5 cases were referred from other hospital. The need for hysterectomy was avoided in all the cases. Uterine and ovarian vessel ligation was done in 4 cases and Gunashila's universal

1. Assistant Professor, Department of Obstetrics and Gynecology, Surat Municipal Institute of Medical Education and Research (SMIMER), Surat

2. Assistant Professor, Department of Community Medicine, Surat Municipal Institute of Medical Education and Research (SMIMER), Surat.

3. Postgraduate resident, Department of Community Medicine, Surat Municipal Institute of Medical Education and Research (SMIMER), Surat. 
circumferential sutures were taken in 3 cases in addition to Hayman suture. The procedure was done with Vicryl No-1 (Polyglactin) in all the cases.

The procedure for Hayman suture technique was as follows:

1. The patient was given appropriate anesthesia and catheterized.

2. The abdomen was opened by an appropriate sized incision or if the patient had a caesarean section, the same incision.

3. On entering the abdomen the uterus was exteriorized and rechecked to identify any bleeding point. If bleeding was diffuse as in uterine atony, coagulopathy, or profuse placental bed bleeding where no obvious bleeding point was observed, then bimanual compression was first applied to assess the potential chances of success of the Hayman suturing technique. The vagina was swabbed to confirm adequate control of the bleeding.

4. If the vaginal bleeding was controlled, a straight needle or a curved needle was used to transfix the uterus from front to back, just above the reflection of the bladder and was then tied at the fundus of the uterus. This can be done as one suture on each side of the uterus or more than one suture if uterus is particularly broad ${ }^{6}$.

\section{Results:}

Age distribution of women is shown in figure 1 , gestational weeks in table 1 , mode of delivery in figure 2 , and type of intervention in table 2 .

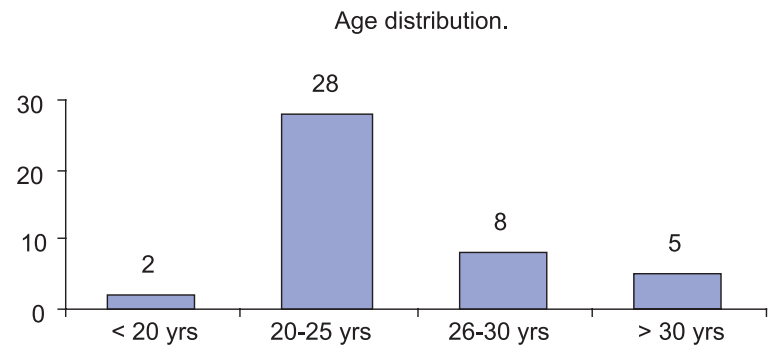

Fig.-1: Age-wise distribution of cases $(n=43)$

Table-I

Gestational age of case ( $n=43)$

\begin{tabular}{lc}
\hline Gestational age & Number $(\%)$ \\
\hline Term & $35(81.4)$ \\
Pre-term & $8(18.6)$ \\
\hline
\end{tabular}

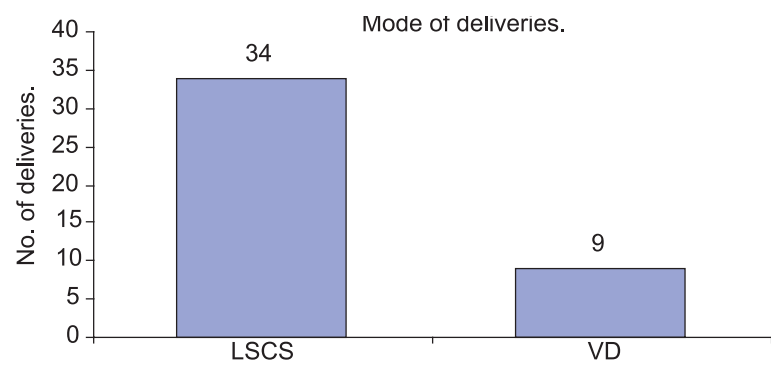

Fig.-2: Mode of delivery $(n=43)$

Table-II

Type of intervention

\begin{tabular}{lc}
\hline Type of intervention & Number (\%) \\
\hline Hayman suture & $36(83.7)$ \\
$\begin{array}{l}\text { Hayman suture + (ovarian + } \\
\text { uterine) vessel ligation }\end{array}$ & $4(9.3)$ \\
$\begin{array}{l}\text { Hayman suture + (ovarian + uterine) } \\
\text { vessel ligation + Gunashila's } \\
\text { circumferential suture }\end{array}$ & $3(7)$ \\
\hline
\end{tabular}

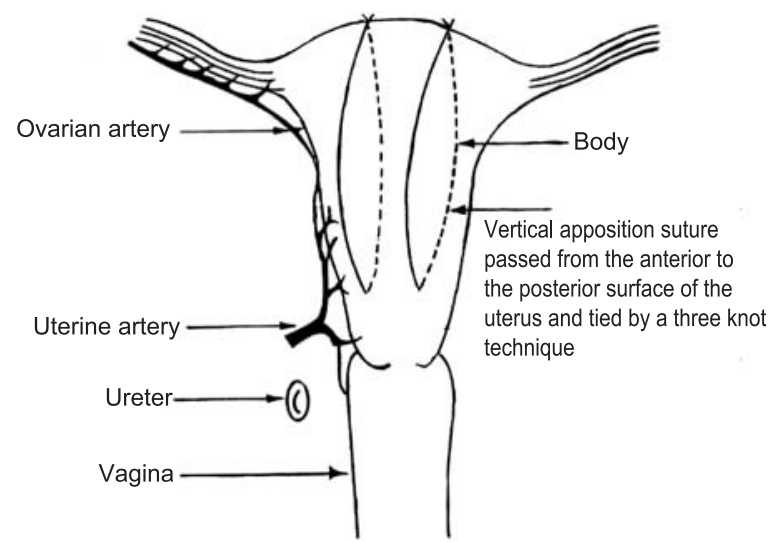

Fig.-3: shows the photograph of Hayman suture taken in a case of atonic PPH.

The Hayman suture was attempted in a total of 43 cases from April, 2007 to March, 2010. All the 43 cases were performed by consultants familiar with the technique. Bleeding was controlled in all the cases with Hayman sutures thus averting hysterectomy. Four (9.3\%) required additional uterine and ovarian vessel ligation. Three (7\%) required additional Gunashila's universal circumferential sutures. All the cases had evidence of uterine atony which responded poorly to conventional uterotonics. The estimated blood loss ranged from $750 \mathrm{ml}$ to $5000 \mathrm{ml}$. All the patients had an uneventful post-operative recovery except in one referred case, in whom 
bleeding was controlled with Hayman sutures but later she developed septicemia plus Acute Respiratory Distress Syndrome (ARDS) and died on $21^{\text {st }}$ postpartum day.

\section{Discussion:}

Christopher B-Lynch's original case series of five patients underwent the B-Lynch's procedure for massive PPH as conventional uterotonic agents proved ineffective ${ }^{4}$. In 2002, Hayman placed two vertical sutures on each side of the fundus of the uterus in three patients with $\mathrm{PPH}$ without performing a hysterotomy ${ }^{7}$. The procedure was successful in preserving the uterus and hence fertility. Although uterine atony is often the indication for the use of the compression sutures, it has been shown in many case reports that the suture is also useful in controlling bleeding in cases of placenta previa and placenta accreta ${ }^{7}$.

Various suture materials have been tried, including Vicryl (polyglactin910), Dexon (polyglycolic acid), PDS (polydioxanone), prolene (monofilament polypropylene) and nylon. It is believed that the ideal suture should be strong, monofilament (to minimize possible trauma to the friable tissue of the atonic uterus), quickly absorbed, and mounted on a large curved or on a straight needle. Ideally the suture needs to maintain the tensile strength for 48-72 hours. In our series of 43 cases, Vicryl was used in all cases. There were no subsequent long term complications encountered in these patients.

There have been isolated reports of adverse consequences after B-Lynch application. In 2004, Grotegut et al reported one case of erosion of a BLynch suture through the uterine wall, in a 19 year old primi para ${ }^{8}$. Partial ischemic necrosis of the uterus occurring 24 hours after the procedure has also been reported in a 26 year old primi para, who underwent an emergency caesarean section for fetal distress followed by B-Lynch suture ${ }^{9}$. The effect of the erosion on future fertility and labor remains unknown. Despite this, many patients on long term follow up have demonstrated resumption of normal menstrual periods and normal reproductive health ${ }^{10}$.

Long term complications such as, formation of bowel adhesion have also been reported ${ }^{7}$.

Comparing B-Lynch suture with Hayman suture, BLynch is time consuming and there is bleeding from uterus due to multiple bites. Uterine opening is avoided with Hayman suture. Cervical stenosis, haematometra, partial necrosis and sloughing of uterine wall have been documented by various surgeons as a complication of original B-Lynch suture technique. Since modified B-Lynch brace sutures is a newer technique, and most of the studies are based on case reports and are without proper controlled studies, more controlled studies are required before it is accepted as a standard method 6 .

In our series, none of the patients had any known adverse outcome till date. Our series of 43 patients illustrate the usefulness of the Hayman procedure in the management of intractable $\mathrm{PPH}$, thus avoiding hysterectomy. To date, we have no further data of continued fertility in patients whose uterus was preserved. There is no randomized controlled data comparing Hayman procedure to other methods of haemostasis for $\mathrm{PPH}$, and it is unlikely that such data would ever be forthcoming, given that $\mathrm{PPH}$ is often unanticipated and occurs under urgent or life threatening situations, thereby rendering randomization and the process of controlling for variables extremely is difficult, if not impossible to implement and ethically questionable ${ }^{10}$.

\section{Conclusion:}

Our initial series of cases of atonic PPH treated with Hayman procedure shows that it is an effective method of controlling PPH. The Hayman suture has the advantage of being applied easily and rapidly. It should be attempted as early as possible in order to maximize its success and prophylactic application should be considered in patients at high risk. Application of a Hayman sutures should be taught to all trainees and registrars in obstetrics. Its relative simplicity and ease of application, its life saving potential, relative safety, and above all, its capacity for preserving the uterus, makes it the recommended procedure of choice if conservative measures do not control PPH, and should be attempted before any radical surgery is considered. It does not require any extra skill and even a resident doctor or a junior doctor can perform it.

\section{References:}

1. Drife J .Management of primary postpartum hemorrhage. BJOG 1997; 104: 275-7.

2. Coombs CA, Murphy El, Laros RK. Factors associated with postpartum hemorrhage with vaginal birth. Obst et Gynecol 1991; 77: 69-76. 
3. Kaurtz AM, Hugues JM, Grinies DA et al. Causes of maternal mortality in United States. Obstet Gynecol 1985; 65: 605-82.

4. B-Lynch C, Coker A, Lawal AH, AbuJ, CowenMJ. The B-Lynch surgical technique for control of massive postpartum hemorrhage: an alternative to hysterectomy? Five cases reported. Br j Obstet Gynaecol 1997; 104: 372-5.

5. Ghezzi F, Cromi A, Uccella S,Raio L, Bolis P, Surbek D. The Hayman technique: a simple method to treat postpartum hemorrhage. BJOG 2007; 114: 362-5.

6. Ghodake VB, Pandit SN, Umbardand SM.Role of modified B-Lynch suture in modern day management of atonic post partum hemorrhage.
JOGC Bombay Hospital Journal, 2008; 50: 206- 207.

7. Hayman RG, Arulkumaran S, Steer PJ. Uterine compression sutures; surgical management of postpartum hemorrhage. Obstet Gynaecol 2002; 99: 2-6.

8. Grotegut CA, Larsen FW, Jones MR, Livingston E. Erosion of a B-Lynch suture through the uterine wall: a case report. J Reprod Med 2004; 49: 849-52.

9. Joshi MV, Shrivastava M. Partial ischaemic necrosis of the uterus following a uterine brace compression suture. BJOG 2004; 111: 279-80.

10. Wohlmuth CT, Gumbs J, Quebral-Ivie. B-Lynch suture a case series. Int Fertil Womens Med 2005; 50:164-73. 